\title{
NEURODEVELOPMENTAL VERSUS NEURODEGENERATIVE MODEL OF SCHIZOPHRENIA AND BIPOLAR DISORDER: COMPARISON WITH PHYSIOLOGICAL BRAIN DEVELOPMENT AND AGING
}

\author{
Massimiliano Buoli, Marta Serati, Alice Caldiroli, Laura Cremaschi \& Alfredo Carlo Altamura \\ Department of Psychiatry, University of Milan, Fondazione IRCCS Ca' Granda Ospedale Maggiore Policlinico, Milan, Italy \\ received: 27.6.2016; \\ revised: 17.10.2016; \\ accepted: 3.1.2017
}

\section{SUMMARY}

Available data support a contribution of both neurodevelopmental and neurodegenerative factors in the etiology of schizophrenia $(\mathrm{SCH})$ and bipolar disorder (BD). Of note, one of the most important issue of the current psychiatric research is to identify the specific factors that contribute to impaired brain development and neurodegeneration in $\mathrm{SCH}$ and $\mathrm{BD}$, and especially how these factors alter normal brain development and physiological aging process. Our hypothesis is that only specific damages, taking place in precise brain development stages, are associated with future SCH /BD onset and that neurodegeneration consists of an acceleration of brain aging after SCH /BD onset. In support of our hypothesis, the results of the present narrative mini-review shows as neurodevelopmental damages generally contribute to neuropsychiatric syndromes (e.g. hypothyroidism or treponema pallidum), but only some of them are specifically associated with adult SCH and BD (e.g. toxoplasma or substance abuse), particularly if they happen in specific stages of brain development. On the other hand, cognitive impairment and brain changes, associated with long duration of SCH /BD, look like what happens during aging: memory, executive domains and prefrontal cortex are implicated both in aging and in SCH /BD progression. Future research will explore possible validity of this etiological model for SCH and BD.

Key words: schizophrenia (SCH) - bipolar disorder (BD) - neurodevelopment - neurodegeneration - aging

\section{INTRODUCTION}

Neurodegenerative and neurodevelopmental hypothesis are actually two alternative theories to explain schizophrenia (SCH) and bipolar disorder (BD) etiology. Neurodevelopmental hypothesis suggests that a disruption of brain development during early life is responsible for later onset of symptoms, while neurodegenerative hypothesis highlights the "negative" effects of illness (Altamura et al. 2014). Available data support a contribution of both neurodevelopmental/ neurodegenerative processes in the etiology of these disorders, but the real question is to understand whether specific versus generic damage of brain neurodevelopment is associated later with $\mathrm{SCH} / \mathrm{BD}$ phenotype and whether neurotoxic effects of illness differ significantly from normal brain aging (Schnack et al. 2016). Our hypothesis is that only specific damages, taking places in precise neurodevelopment stages contribute to bipolar/ schizophrenic phenotype and that, after psychopathological onset, there is an acceleration of brain aging (Figure 1). First objective of the present paper is, therefore, to describe the main evidence in support of neurodevelopmental versus neurodegenerative model of $\mathrm{SCH} / \mathrm{BD}$. Second objective is to compare etiological models of $\mathrm{SCH} / \mathrm{BD}$ with physiological neurodevelopment and aging in order to identify the specific risk factors associated with a diagnosis of SCH/BD.

\section{METHODS}

A search of articles on MEDLINE, PsycINFO, Isi Web of Knowledge, Medscape was performed in order to obtain a narrative mini-review about the hypothetical etiology of $\mathrm{SCH}$ and $\mathrm{BD}$ (neurodevelopmental versus neurodegenerative model) compared to physiological brain development and aging. The words "SCH", "BD", "physiology" have been associated with "neurodevelopment", neurodegeneration" and "aging". A manual selection of papers was then performed in order to consider the most recent and relevant articles (controlled studies, review or meta-analyses) concerning with the topic of the present article. Only papers in English were included. This search covered findings from 2002 and 2016, with last check in March 2016. Exclusion criteria included animal studies or samples of patients with a diagnosis different from $\mathrm{SCH}$ and $\mathrm{BD}$

\section{PHYSIOLOGICAL BRAIN DEVELOPMENT VERSUS NEURODEVELOPMENTAL ABNORMALITIES}

Three basic stages are recognized in brain development:

- pregnancy: development of neurons and their connections; 


\section{Pregnancy Childhood Adolescence Onset of Illness Aging}

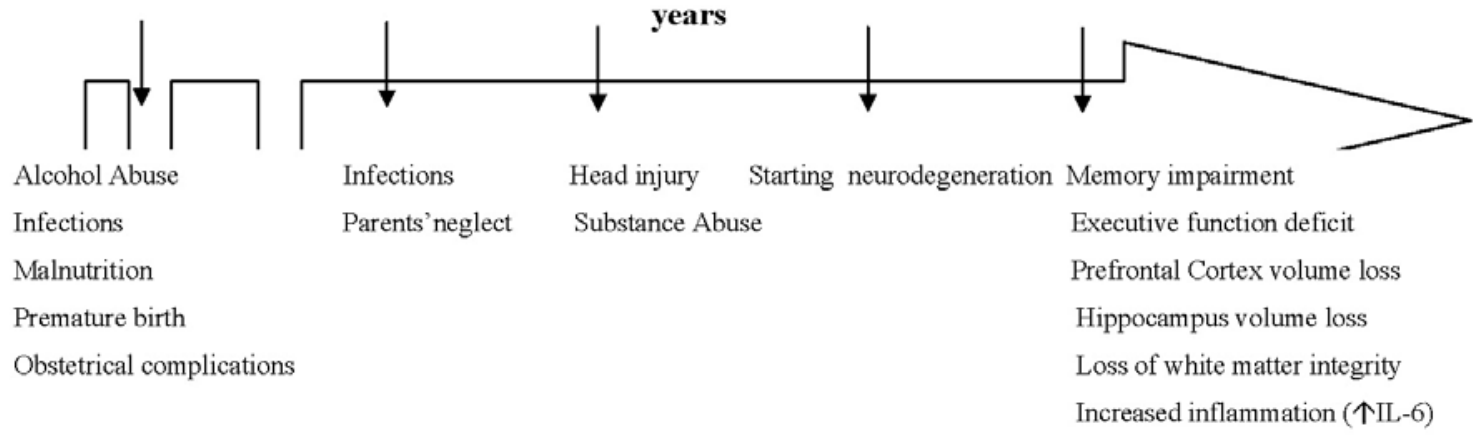

Figure 1. Biological history of Schizophrenia and Bipolar Disorder

Table 1. Main factors that have been associated with brain neurodevelopment abnormalities and clinical symptoms

\begin{tabular}{lll}
\hline Pregnancy & Childhood & Adolescence \\
\hline Alcohol abuse & Infectious Agents (e.g. Toxoplasma, & Head injuries \\
Chromosome abnormalities & Cytomegalovirus, Streptococcus & Substance Abuse \\
Drug Abuse & Pyogenes-PANDAS) & \\
Environmental Chemicals (e.g. DDT) & Environmental Chemicals (e.g. DDT) & \\
Genetic predisposition & Dysmetabolisms (e.g. phenylketonuria) & \\
Hypothyroidism & General anestetics & \\
Infectious Agents (e.g. Rubella, Influenza, & Malnutrition & \\
Herpes Simplex, & Parents’ neglect & \\
Cytomegalo Viruses; Toxoplasma; & \\
Treponema Pallidum) & \\
Malnutrition & \\
Obstetrical complications & \\
Premature birth & \\
\hline
\end{tabular}

Note: in italic the factors associated with increased risk of schizophrenia/bipolar disorder

PANDAS: pediatric autoimmune neuropsychiatric disorders; DDT: dichlorodiphenyltrichloroethane

- 0-6 years: gray matter growth (at the age of six human brain presents $95 \%$ of the weight of the adult brain and it has the metabolic peak demand);

- 6 years-early adulthood: development of white matter and connectivity (Groeschel et al. 2010).

Each of these phases is particularly sensitive to specific damages that can affect the brain development (Table 1). However, only some of these "toxic" factors are associated with the development of a future psychiatric condition, while others determine unspecific neurological syndromes. In the following paragraphs factors that have been specifically associated with an increased risk of $\mathrm{SCH} / \mathrm{BD}$ are described.

Infections of influenza, rubella, herpes simplex viruses and toxoplasma gondii during pregnancy have been all associated with a higher risk of developing $\mathrm{SCH}$ and $\mathrm{BD}$ (influenza virus and toxoplasma gondii) in the offspring (Altamura et al. 2014; Brown 2011; Parboosing et al. 2013). With regard to influenza virus, Parboosing and collaborators found nearly 4-fold increase in the risk of $\mathrm{BD}(\mathrm{OR}=3.8295 \% \mathrm{CI}=1.58-9.24)$ after exposure to maternal influenza at any time during pregnancy (Parboosing et al. 2013). A study demonstrated that malnutrition during pregnancy determines 2-fold increased risk of SCH in a sample of 52-011 newborns $(\mathrm{RR}=2.2595 \% \quad \mathrm{CI}=2.00-2.52)$ (Xu et al. 2009). In addition, children born from 32 to 36 weeks' gestation were found to be more likely to have non-affective psychosis $(\mathrm{OR}=1.395 \%$ CI 1.1-1.7) and BD (OR=2.7 95\% CI 1.6-4.5) (Nosarti et al. 2012). Also prenatal alcohol exposure seems to increase the risk of BD (O'Connor et al. 2002). Finally obstetric complications were found to increase the risk of future $\mathrm{SCH}$ and $\mathrm{BD}$, although less evidence is reported for BD (Cannon et al. 2002, Scott et al. 2006).

In childhood low socio-economic status, with related poor nutrition, and parents' neglect, infections (toxoplasma gondii and cytomegalovirus) are all associated with increased risk of future SCH (Brown 2011). Similarly, a recent paper highlighted as childhood emotional neglect is significantly associated with $\mathrm{BD}$ in adulthood (Watson et al. 2013)

Among adolescents, substance abuse (more robust evidence for cannabis) represents the most important non-genetic factor associated with an increased risk of $\mathrm{SCH}$ or $\mathrm{BD}$, particularly in subjects with genetic predisposition (De Hert et al. 2011). A recent largesample follow-up study found that people, who had 
suffered from head injuries $(\mathrm{N}=113906)$, had an increased risk of a subsequent diagnosis of $\mathrm{SCH}$ (incidence rate ratio $[\mathrm{IRR}]=1.65,95 \% \mathrm{CI}=1.55-1.75$ ), $\mathrm{BD}(\mathrm{IRR}=1.28,95 \% \mathrm{CI}=1.10-1.48)$, unipolar depression $(\mathrm{IRR}=1.5995 \% \mathrm{CI}=1.53-1.65)$ and organic mental disorders $(\mathrm{IRR}=4.39$, 95\% $\mathrm{CI}=3.86-4.99)$. Individuals with psychiatric diagnoses before injury, including substance use disorders, had been excluded (Orlovska et al. 2014).

\section{AGING VERSUS NEURODEGENERATION}

In normal brain aging, reduced white matter integrity and global cognitive decline (especially in memory and executive domains) have been documented by a number of data (Glahn et al. 2013). The speed and intensity of these processes are partly influenced by genetic disposition (Glahn et al., 2013). In addition, global brain metabolism declines with age, particularly in left inferior frontal junction (Chètelat et al. 2013), anterior cingulate/medial prefrontal cortex, dorsomedial thalamus and subgenual cingulate/basal forebrain (Pardo et al. 2007). Atrophy of hippocampus and prefrontal cortices are also documented as well as an increase of inflammation and production of free radicals (Buzynska et al. 2012). Interleukin 6 (IL-6) was found to increase with aging and higher levels of this cytokine have been associated with an increased risk of both cardiovascular events and non-cardiovascular death (Akbaraly et al. 2013). The inflammatory hypothesis of schizophrenia has recently regained interest also in light of the several data describing the efficacy of some components, such as aspirin or celecoxib, on symptom severity (Sommer et al. 2014, Andrade 2016). Similarly TNF-alpha inhibitors have been proposed for the treatment of depression (Schmidt et al. 2014, Kaster et al. 2016). These findings suggest a potential role of the immune system in the pathogenesis of main psychiatric disorders, with important implication for future treatment options of schizophrenia (Girgis et al. 2014).

Neurotoxic effects of SCH and BD predominantly affect some specific brain areas as an effect of immune dysregulation and with the result of a precise cognitive dysfunction. Similarly to normal aging, the most compromised brain areas in patients with $\mathrm{SCH}$ and BD include prefrontal cortex and hippocampus (Brown et al. 2011). In addition, memory and executive functions are highly compromised in SCH and BD patients particularly in individuals with long duration of illness (Buoli et al. 2014). Finally, increased IL-6 has been proposed as a common biomarker of immune dysregulation in SCH and BD (Altamura et al. 2014). In light of these considerations, a very recent publication stated that the net result of biological changes, which are associated with $\mathrm{SCH}$ and $\mathrm{BD}$, is an accelerated brain aging (Koutsoluleris et al. 2014)

\section{DISCUSSION}

As mentioned above, a limited number of negative effects during neurodevelopment are specifically associated with $\mathrm{SCH}$ and $\mathrm{BD}$ onset in adulthood. In addition, the damage has to happen in specific stages of neurodevelopment. For example, a number of data indicate that substance abuse (particularly cannabis) during adolescence increases the risk of SCH and BD (De Hert et al. 2011), while mothers' substance abuse during pregnancy may produce neurological syndromes in the future unborn, but it not certain the association with SCH and BD. Similarly, influenza epidemics in mothers increase the risk of SCH in offspring, but there is no effect of this virus in childhood or adolescence, while cytomegalovirus infections increase the risk of schizophrenia only if they happen during childhood (Brown 2011). In contrast, toxoplasma infections are associated with an increased risk of SCH and BD both if the infections occur during pregnancy or childhood (Brown, 2011). These considerations have two important clinical implications: 1) specific risk factors associated with a significant increased risk of SCH or $\mathrm{BD}$ can be reduced 2) high-risk adolescents, who have been exposed during their life to the cited harmful factors, may be more easily identified.

With regard to neurodegeneration, an increasing number of evidence indicates that $\mathrm{SCH}$ and $\mathrm{BD}$ lead to a premature brain aging so that main target of treatment should be the slowdown of biological changes associated with these conditions (Koutsoluleris et al. 2014; Altamura et al. 2015).

Finally, further data about the etiology of these disorders will confirm if the model of illness, proposed in this paper, may be definitively recognized as valid.

In conclusion, both neurodevelopment abnormalities and neurodegenerative aspects would appear as valid models to explain the etiology of SCH/BD. The study of physiological neurodevelopment and normal brain aging may help researchers to identify specific risk factors associated with $\mathrm{SCH} / \mathrm{BD}$ diagnoses.

This paper presents some limitations: patients' habits such as smoking, eating and drugs, have to be considered as factors that can impact cytokine leves; furthermore, it has been described that some drugs may increase C-reactive protein levels and may induce fever via cytokines, leading to elevated IL- 6 and TNF- $\alpha$ (Štuhec 2013). In addition this paper is not a systematic review, being the purpose of the present article to give a concise overview of the etiological models of schizophrenia and bipolar disorder, and of risk factors associated with these mental disorders.

\section{Acknowledgements: None.}

Conflict of interest: None to declare. 


\section{Contribution of individual authors:}

Massimiliano Buoli, Marta Serati: wrote the manuscript; Alice Caldiroli, Laura Cremaschi: searched articles; Alfredo Carlo Altamura: reviewed the paper.

\section{References}

1. Akbaraly TN, Hamer M, Ferrie JE, Lowe G, Batty GD, Hagger-Johnson $G$ et al.: Chronic inflammation as a determinant of future aging phenotypes. CMAJ 2013; 185:E763-70

2. Altamura AC, Buoli $M$ \& Pozzoli S: Role of immunological factors in the pathophysiology and diagnosis of bipolar disorder: comparison with schizophrenia. Psychiatry Clin Neurosci 2014; 68:21-36.

3. Altamura AC, Serati $M \&$ Buoli M: Is duration of illness really influencing outcome in major psychoses? Nord $J$ Psychiatry 2015; 69:403-17.

4. Andrade C: Anti-inflammatory strategies in the treatment of schizophrenia. Expert Rev Clin Pharmacol 2016; 9:161-3.

5. Brown AS: The environment and susceptibility to schizophrenia. Prog Neurobiol 2011; 93:23-58.

6. Brown GG, Lee JS, Strigo IA, Caligiuri MP, Meloy MJ \& Lohr J: Voxel-based morphometry of patients with schizophrenia or bipolar I disorder: a matched control study. Psychiatry Res 2011; 194:149-56.

7. Buoli M, Caldiroli A, Caletti E, Zugno E \& Altamura AC: The impact of mood episodes and duration of illness on cognition in bipolar disorder. Compr Psychiatry 2014; 55:1561-6.

8. Cannon M, Jones PB \& Murray RM: Obstetric complications and schizophrenia: historical and meta-analytic review. Am J Psychiatry 2002; 159:1080-92.

9. Chételat G, Landeau B, Salmon E, Yakushev I, Bahri MA, Mézenge F et al.: Relationships between brain metabolism decrease in normal aging and changes in structural and functional connectivity. Neuroimage 2013; 76:167-77.

10. De Hert M, Wampers $M$, Jendricko T, Franic T, Vidovic $D$, De Vriendt $N$ et al.: Effects of cannabis use on age at onset in schizophrenia and bipolar disorder. Schizophr Res 2011; 126:270-6.

11. Girgis RR, Kumar SS \& Brown AS: The cytokine model of schizophrenia: emerging therapeutic strategies. Biol Psychiatry 2014; 75:292-9.

12. Glahn DC, Kent JW Jr, Sprooten E, Diego VP, Winkler AM, Curran JE et al.: Genetic basis of neurocognitive decline and reduced white-matter integrity in normal human brain aging. Proc Natl Acad Sci USA 2013; 110:19006-11.
13. Groeschel S, Vollmer B, King MD \& Connelly A: Developmental changes in cerebral grey and white matter volume from infancy to adulthood. Int J Dev Neurosci 2010; 28:481-489.

14. Kaster MP, Moretti M, Cunha MP \& Rodrigues AL: Novel approaches for the management of depressive disorders. Eur J Pharmacol 2016; 771:236-40.

15. Koutsouleris N, Davatzikos C, Borgwardt S, Gaser C, Bottlender R, Frodl $T$ et al.: Accelerated brain aging in schizophrenia and beyond: a neuroanatomical marker of psychiatric disorders. Schizophr Bull 2014; 40:1140-53.

16. Nosarti C, Reichenberg A, Murray RM, Cnattingius S, Lambe MP, Yin L et al.: Preterm birth and psychiatric disorders in young adult life. Arch Gen Psychiatry 2012; 69:E1-8.

17. O'Connor MJ, Shah B, Whaley S, Cronin P, Gunderson B \& Graham J: Psychiatric illness in a clinical sample of children with prenatal alcohol exposure. Am J Drug Alcohol Abuse 2002; 28:743-54.

18. Orlovska S, Pedersen MS, Benros ME, Mortensen PB, Agerbo $E$ \& Nordentoft M: Head injury as risk factor for psychiatric disorders: a nationwide register-based followup study of 113,906 persons with head injury. Am J Psychiatry 2014; 171:463-9.

19. Parboosing R, Bao Y, Shen L, Schaefer CA \& Brown AS: Gestational influenza and bipolar disorder in adult offspring. JAMA Psychiatry 2013; 70:677-85.

20. Pardo JV, Lee JT, Sheikh SA, Surerus-Johnson C, Shah H, Munch KR et al.: Where the brain grows old: decline in anterior cingulate and medial prefrontal function with normal aging. Neuroimage 2007;35:1231-7.

21. Schmidt FM, Kirkby KC \& Himmerich H: The TNF-alpha inhibitor etanercept as monotherapy in treatment-resistant depression - report of two cases. Psychiatr Danub 2014; 26:288-90.

22. Schnack HG, van Haren NE, Nieuwenhuis M, Hulshoff Pol HE, Cahn W \& Kahn RS: Accelerated brain aging in schizophrenia: a longitudinal pattern recognition study. Am J Psychiatry 2016; 173:607-16.

23. Scott J, McNeill Y, Cavanagh J, Cannon M \& Murray R: Exposure to obstetric complications and subsequent development of bipolar disorder: Systematic review. $\mathrm{Br} \mathrm{J}$ Psychiatry 2006; 189:3-11.

24. Sommer IE, van Westrhenen R, Begemann MJ, de Witte $L D$, Leucht $S$ \& Kahn RS: Efficacy of anti-inflammatory agents to improve symptoms in patients with schizophrenia: an update. Schizophr Bull 2014; 40:181-91.

25. Štuhec M: Clozapine-induced elevated C-reactive protein and fever mimic infection. Gen Hosp Psychiatry 2013; 35:680.e5-6.

26. Xu MQ, Sun WS, Liu BX, Feng GY, Yu L, Yang L et al.: Prenatal malnutrition and adult schizophrenia: further evidence from the1959-1961 Chinese famine. Schizophr Bull 2009; 35:568-76.

\section{Correspondence:}

Assistant Professor Massimiliano Buoli, MD, Phd

Department of Psychiatry, University of Milan

Fondazione IRCCS Ca'Granda Ospedale Maggiore Policlinico

via Francesco Sforza 35, 20122, Milan, Italy

E-mail:massimiliano.buoli@hotmail.it 